\title{
Changes in the Intra-Amniotic Pressure following Transabdominal Amnioinfusion during Pregnancy
}

\author{
Daisuke Katsura $^{\text {a, b }}$ Yuichiro Takahashia Shigenori Iwagaki ${ }^{a} \quad$ Rika Chiaki $^{a}$ \\ Kazuhiko Asai $^{\mathrm{a}}$ Masako Koike ${ }^{\mathrm{a}}$ Takashi Murakami $^{\mathrm{b}}$ \\ aDepartment of Fetal-Maternal Medicine, Nagara Medical Center, Gifu, Japan; bepartment of Obstetrics and \\ Gynecology, Shiga University of Medical Science Hospital, Otsu, Japan
}

\section{Keywords}

Amnioinfusion - Amniotic fluid index · Complication ·

Pressure

\begin{abstract}
Objective: The aim of the article was to investigate the changes in intra-amniotic pressure following transabdominal amnioinfusion during pregnancy. Design: This retrospective study included 19 pregnant women who underwent transabdominal amnioinfusion during pregnancy to relieve umbilical cord compression and improve the intrauterine environment or to increase the accuracy of ultrasonography. Materials and Methods: We measured and analyzed the changes in intra-amniotic pressure, single deepest pocket, and the amniotic fluid index before and after amnioinfusion. We also determined the incidence of maternal or fetal adverse events, such as preterm premature rupture of membranes, preterm delivery, fetal death within $48 \mathrm{~h}$, placental abruption, infection, hemorrhage, and peripheral organ injury. Results: A total of 41 amnioinfusion procedures were performed for 19 patients. The median gestational age during the procedure was 24.3 weeks. The median volume of the injected amniotic fluid was $250 \mathrm{~mL}$. The median single deepest pocket and amniotic fluid index after amnioinfusion
\end{abstract}

were significantly higher than those before amnioinfusion (4.0 cm vs. $2.65 \mathrm{~cm} ; p<0.001$ and $13.4 \mathrm{~cm}$ vs. $6.0 \mathrm{~cm} ; p<$ $0.001)$. However, the median (range) intra-amniotic pressure after amnioinfusion was not significantly different compared to that before amnioinfusion $(11 \mathrm{~mm} \mathrm{Hg}$ vs. $11 \mathrm{~mm} \mathrm{Hg}$; $p=0.134)$. Maternal or fetal adverse events were not observed following amnioinfusion. Conclusion: Intra-amniotic pressure remained unchanged following amnioinfusion. The complications associated with increased intra-amniotic pressure are not likely to develop if the amniotic fluid index and/or single deepest pocket remains within the normal range after amnioinfusion. Studies of groups with and without complications are warranted to clarify the relationship between the intra-amniotic pressure and incidence of complications.

(c) 2021 The Author(s).
Published by S. Karger AG, Basel

\section{Introduction}

Amnioinfusion (AI) is effective for the treatment of variable decelerations in the first stage of labor $[1,2]$. It is also considered effective for the treatment of umbilical cord compression in fetal growth restriction with oligohydramnios [3], idiopathic oligohydramnios [4], or um- karger@karger.com www.karger.com/bmh

Karger $\stackrel{\text { ' }}{5}$

GOPEN ACCESS
(C) 2021 The Author(s)

Published by S. Karger AG, Basel

This is an Open Access article licensed under the Creative Commons Attribution-NonCommercial-4.0 International License (CC BY-NC) (http://www.karger.com/Services/OpenAccessLicense), applicable to the online version of the article only. Usage and distribution for commercial purposes requires written permission.
Correspondence to:

Daisuke Katsura, katsuo14@belle.shiga-med.ac.jp 


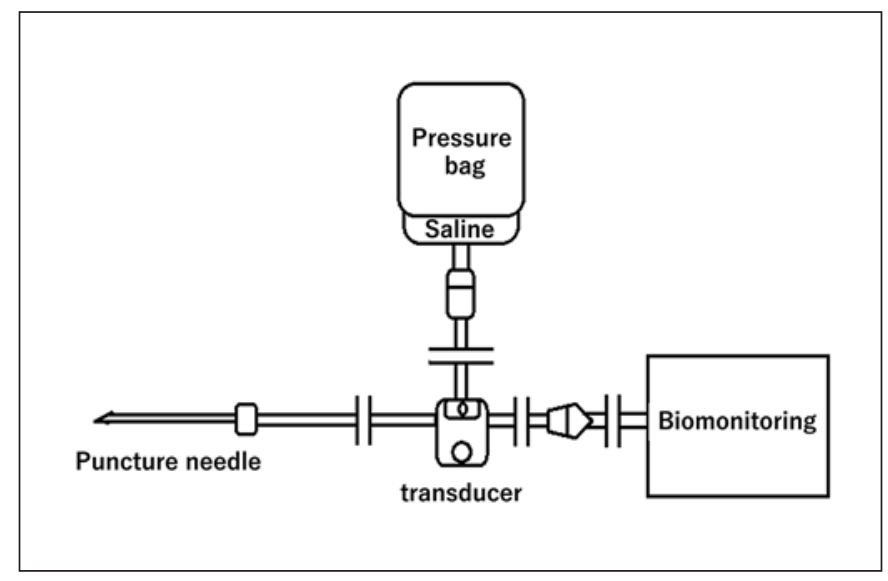

Fig. 1. Circuit including a silicon strain-gauge transducer (DX300; Nihon Kohden Corporation, Tokyo, Japan) for measuring pressure.

bilical cord compression without oligohydramnios [5] during pregnancy. Additionally, it is used to increase the ultrasonographic accuracy in identifying structural anomalies in patients with oligohydramnios $[2,6]$. Although the use of therapeutic AI during pregnancy prior to parturition has not yet been established, transabdominal $\mathrm{AI}$ is expected to be a new intervention for umbilical cord compression and oligohydramnios. However, indications for its use should be carefully selected because adverse events associated with AI have been previously reported [7].

Reported adverse events occurring due to transabdominal AI include miscarriage, premature rupture of membranes, preterm labor, placental abruption, chorioamnionitis, fetal trauma, and uterine perforation [7]. Previous studies reported the relationship between high intra-amniotic pressure and polyhydramnios and between high intra-amniotic pressure and maternal symptoms such as abdominal distension and dyspnea, threatened premature labor, and preterm labor $[8,9]$. An increase in the intra-amniotic pressure due to AI may lead to the development of complications, such as premature rupture of membranes and preterm labor. We hypothesized that the intra-amniotic pressure remains unchanged if the amniotic fluid index (AFI) after AI is within the normal range; however, this has not been systematically examined. This study aimed to investigate the change in the intra-amniotic pressure after AI. To the best of our knowledge, this is the first study to systematically report the changes in intra-amniotic pressure following transabdominal AI.

Intra-Amniotic Pressure following Amnioinfusion

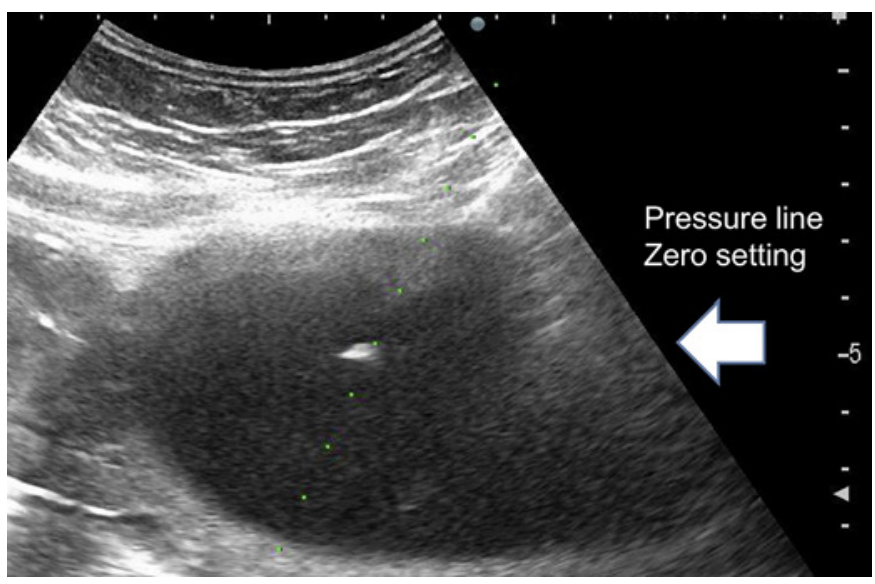

Fig. 2. Ultrasound and zero pressure-level line setting.

\section{Materials and Methods}

This study was approved by the Institutional Review Board of Nagara Medical Center (18-6). In this study, we included pregnant women who underwent transabdominal AI during pregnancy at the Department of Fetal-Maternal Medicine, Nagara Medical Center, between April 2017 and September 2018. Written informed consent was obtained from all patients in whom AI was indicated.

The intra-amniotic pressure was measured before and after the procedure. A saline-filled line was attached to the hub of the needle at one end and to a silicon strain-gauge transducer (DX-300; Nihon Kohden Corporation, Tokyo, Japan) at the other end (shown in Fig. 1). The pressures were measured at the needle tip level (shown in Fig. 2), as reported previously [10, 11], if they were stable for $10 \mathrm{~s}$ without uterine contractions.

AI was indicated for the relief of cord compression in cases of umbilical cord compression, as confirmed by the sandwich sign or by oligohydramnios on ultrasonography and variable decelerations on heart rate monitoring. It was also performed to improve the intrauterine environment or ultrasonographic accuracy in cases involving oligohydramnios. The sandwich sign refers to the ultrasonographic finding of the umbilical cord sandwiched between 2 layers of the placenta or between the placenta and the fetus, without amniotic fluid present around the umbilical cord, which indicates local oligohydramnios [5]. As the sandwich sign alone does not necessarily reflect umbilical cord compression, AI was performed when variable decelerations were confirmed more than once within 40 $\mathrm{min}$ and were reproducible during heart rate monitoring accompanied with the sandwich sign on ultrasonography. Tocolysis was performed by the infusion of ritodrine hydrochloride $(50 \mu \mathrm{g} / \mathrm{min})$ or magnesium sulfate $(20 \mathrm{~mL} / \mathrm{h})$ during the AI procedure to prevent uterine contractions. Experimental obstetricians performed AI using a 21-gauge percutaneous transhepatic cholangiography needle under ultrasound guidance, with the help of a needle adapter. First, $100 \mathrm{~mL}$ of warm saline $(100 \mathrm{~mL})$ was infused via drip infusion or manually if the tip of the puncture was confirmed to have an amniotic fluid cavity; if necessary, additional warm saline was infused 
Table 1. Basic characteristics and outcomes of patients who underwent $\mathrm{Al}(n=19)$

\begin{tabular}{ll}
\hline Variable & Measure $(n=19)$ \\
\hline Age, years & $33(21-40)$ \\
Primipara, $\%(n / N)$ & $36.8(7 / 19)$ \\
Hypercoiled cord, \% $(n / N)$ & $63.2(12 / 19)$ \\
Fetal structural anomaly, \% $(n / N)$ & $26.3(5 / 19)$ \\
Massive subchorionic hematoma, \% $(n / N)$ & $10.5(2 / 19)$ \\
GA at Al, weeks and days & 24 weeks 3 days (19 weeks 3 days-33 weeks 6 days) \\
Injected amniotic fluid volume, $\mathrm{mL}$ & $250(120-400)$ \\
GA at delivery, weeks and days & 34 weeks 3 days $(27$ weeks 0 days-37 weeks 3 days) \\
Cesarean delivery, \% $(n / N)$ & $52.6(10 / 19)$ \\
$Z$ score of birth weight (median $[S D])$ & $-1.1(-3.5-[-0.1])$ \\
Apgar score & $8(3-9)$ \\
$\quad 1$ min & $9(7-9)$ \\
5 min & $7.33(6.96-7.39)$ \\
Umbilical artery pH &
\end{tabular}

The data are presented as median (range) or $\%(n)$ as appropriate. GA, gestational age; SD, standard deviation; Apgar, Appearance, Pulse, Grimace, Activity, and Respiration; Al, amnioinfusion.

until the relief of umbilical cord compression was confirmed by ultrasonography in cases of umbilical cord compression or detection of an AFI $>5 \mathrm{~cm}$. This improves the intrauterine environment or increases the ultrasonographic accuracy in cases of oligohydramnios. After AI, we performed ultrasonography the next day and twice a week until delivery. Continuous maternal and fetal monitoring was conducted to detect symptoms such as frequent uterine contractions, persistent abdominal pain, uterine bleeding, and fetal bradycardia, which might be signs of placental abruption, and low blood pressure, impairment of consciousness, and dyspnea, which might be signs of amniotic fluid embolism. None of the patients had the aforementioned maternal or fetal conditions.

We collected the following data: maternal age, parity, body mass index, gestational age (GA) at AI and delivery, injected amniotic fluid volume, mode of delivery, birth weight, umbilical artery $\mathrm{pH}$, single deepest pocket (SDP), AFI, intra-amniotic pressure before and after AI, and incidence of complications associated with AI. We defined SDP $<2 \mathrm{~cm}$ and AFI $<5 \mathrm{~cm}$ as oligohydramnios and SDP $>8 \mathrm{~cm}$ and AFI $>24 \mathrm{~cm}$ as polyhydramnios $[12,13]$.

The Wilcoxon signed-rank test was used to compare the data. A $p$ value of $<0.05$ was considered statistically significant. The correlation between the volume of the amniotic fluid injected and changes in the intra-amniotic pressure was assessed using Spearman's correlation coefficient, and the correlation between changes in the SDP and AFI and changes in the intra-amniotic pressure was assessed using Pearson's correlation coefficients. Statistical analyses were performed using Easy R (The R Foundation for Statistical Computing, Vienna, Austria) for Windows [14].

\section{Results}

Overall, 41 AI procedures were performed for 19 patients. Thirty-one $(75.6 \%)$ AI procedures were performed for $15(78.9 \%)$ patients with umbilical compression, and
$10(24.4 \%)$ were performed for $4(21.1 \%)$ patients with oligohydramnios. The basic characteristics and clinical outcomes are summarized in Table 1 . The median GA at the time of AI was 24 weeks 3 days of gestation (range, 19 weeks 3 days-33 weeks 6 days); the median volume of the amniotic fluid injected was 250 (range, $120-400$ ) $\mathrm{mL}$, and the median GA at delivery was 34 weeks 2 days (range, 27 weeks 0 days-37 weeks 3 days). Spontaneous vaginal delivery was performed in 9 patients, and cesarean delivery was performed because of a nonreassuring fetal status in 7 patients and breech in 3 patients. The mean $Z$ score for birth weight was -1.1 standard deviation $(-3.5-[-0.1])$, the mean Apgar scores were 8 (range, 3-9) and 9 (7-9) at 1 and $5 \mathrm{~min}$, respectively, and the umbilical artery $\mathrm{pH}$ was 7.33 (6.96-7.39). Although the long-term prognosis could not be evaluated because 3 patients were lost to follow-up, severe brain damage was not observed at the short-term evaluation. Sixteen infants showed normal development at 1 year, and 1 infant needed home oxygen therapy. Of the 19 patients, $12(63.2 \%)$ patients had a hypercoiled cord, $5(26.3 \%)$ patients had fetal structural anomalies, including 3 multicystic dysplastic kidneys, 1 hypoplastic kidney, and 1 omphalocele, and $2(10.5 \%)$ patients had massive subchorionic hematomas (Table 1). In the 2 cases with massive subchorionic hematomas, AIs were performed for the indication of oligohydramnios and umbilical cord compression. No uterine bleeding and hematoma growth were observed after the puncture.

AIs were completed approximately $30 \mathrm{~min}$ after the puncture. The mean frequency of AIs was 1.8 (range, 1-7)
88

Biomed Hub 2021;6:86-91 DOI: $10.1159 / 000519084$
Katsura/Takahashi/Iwagaki/Chiaki/Asai/ Koike/Murakami 
times in 12 cases of hypercoiled cord with umbilical cord compression, 3 (range, 2-6) times in 3 cases of fetal structural anomaly with umbilical cord compression, and 2.6 (range, 2-4) times in 4 cases of fetal structural anomaly and massive subchorionic hematoma with oligohydramnios. No significant difference was observed between these groups. The mean AI interval was 7.2 (range, 2-14) days in cases with a hypercoiled cord with umbilical cord compression, 7.3 (range, 2-12) days in cases of fetal structural anomaly with umbilical cord compression, and 9.9 (range, 7-14) days in cases of fetal structural anomaly and massive subchorionic hematoma with oligohydramnios. No significant difference was observed between these groups. However, it must be considered that the numbers were relatively small for analyzing the relationship between these subgroups.

The median SDP and AFI before AI were 2.65 (range, $0-5.7) \mathrm{cm}$ and 6.0 (range, $0-15) \mathrm{cm}$, and after AI, they increased significantly to 4.0 (range, $2.7-6.1) \mathrm{cm}(p<$ $0.001)$ and 13.4 (range, $2.7-21) \mathrm{cm}(p<0.001)$. However, significant differences were not observed in the median intra-amniotic pressure before and after AI (11 [range, $4-14$ ] $\mathrm{mm} \mathrm{Hg}$ vs. 11 [range, 6-19] mm Hg; $p=0.134$ ). Spearman's correlation coefficient for the volume of the amniotic fluid injected and changes in the intra-amniotic pressure was $r=0.258(p=0.107)$. Pearson's correlation coefficients for changes in the SDP and AFI and changes in the intra-amniotic pressure inspection were $r=0.048$ $(p=0.786)$ and $r=0.029(p=0.865)$.

Regarding the indication of AI, we analyzed the relationship between cases with oligohydramnios and umbilical cord compression. No significant differences were observed in the median intra-amniotic pressure before AI (9 [range, $4-13$ ] $\mathrm{mm} \mathrm{Hg}$ vs. 11 [range, 5-14] $\mathrm{mm} \mathrm{Hg} ; p=0.262$ ) and after AI (10 [range, 7-19] mm Hg vs. 10 [range, 6-14] mm $\mathrm{Hg} ; p=0.264)$, the median volume of amniotic fluid injected (250 [range, 150-300] mm Hg vs. 250 [range, 120-400] $\mathrm{mm} \mathrm{Hg} ; p=0.695$ ), and the median intra-amniotic pressure before and after AI (9 [range, 4-13] mm Hg vs. 10 [range, $7-19$ ] $\mathrm{mm} \mathrm{Hg} ; p=0.131$; and 11 [range, $5-14$ ] $\mathrm{mm} \mathrm{Hg}$ vs. 10 [range, 6-14] mm Hg; $p=0.393$ ) between cases with oligohydramnios and umbilical cord compression.

Maternal or fetal adverse events, such as preterm premature rupture of membranes, delivery and fetal death within $48 \mathrm{~h}$, placental abruption, infection, hemorrhage, and peripheral organ injury, were not observed following AI. Moreover, although mild palpitation and tremor caused by ritodrine hydrochloride administration and mild vascular pain caused by magnesium sulfate administration were observed, no adverse events occurred that required treatment.

Intra-Amniotic Pressure following Amnioinfusion

\section{Discussion}

AI has been reported to be effective in the management of umbilical cord compression and oligohydramnios [1-6]; however, several adverse events are associated with AI [7]. Complications may develop due to the changes in the intra-amniotic pressure during the procedure. Therefore, we measured and compared the intra-amniotic pressure before and after AI. Although the SDP and AFI after AI were significantly higher than those before AI, the intra-amniotic pressure after AI was not significantly different from that recorded before AI. Furthermore, there was no correlation between the volume of the amniotic fluid injected and changes in the SDP and AFI and the intra-amniotic pressure. The low volume of amniotic fluid injected and the SDP and AFI, which was within the normal range after AI, could explain these observations. In addition, we injected $250 \mathrm{~mL}$ (mean) of warm saline at mean 24 weeks 3 days (range, 19 weeks 3 days-33 weeks 6 days) in this study. The injection fluid volume of $250 \mathrm{~mL}$ was not expected to affect the intraamniotic pressure because amniotic fluid volume between 22 and 39 weeks was an average of $777 \mathrm{~mL}$ (range, 302-1,997 mL) [15]. Normal amniotic fluid volume changes during pregnancy [15]; therefore, the injection fluid volume should be determined considering GA.

We hypothesized that the changes in the intra-amniotic pressure during AI may be the cause of the reported complications. In polyhydramnios, the intra-amniotic pressure is high, and amnioreduction is often performed to prevent preterm delivery and relieve maternal symptoms $[8,9]$. We also reported previously that the intraamniotic pressure decreased and reached a plateau during amnioreduction [9]. These results support our hypothesis, and the SDP and AFI after AI should be kept within a normal range because polyhydramnios increases intra-amniotic pressure.

Entire and local oligohydramnios did not influence the change in intra-amniotic pressure following AIs. Leiomyoma and adenomyosis, which cause lesions that can exist within the myometrium, might affect uterine pressure reaction during AIs by limiting the extension of the uterus. In addition, we reported previously that uterine pressure tolerance may vary according to the individual [9]. Therefore, we may have to address changes in the intra-amniotic pressure because the pressure might increase during AI even if there was no polyhydramnios.

Additionally, we observed that the pressure increased when the tip of the needle was in the uterine myometrium or touched or punctured the fetus during AI. This was 
particularly true when the needle punctured the fetus, and the infusion of warm saline was continued, resulting in fetal skin edema. In some cases, it is difficult to ascertain whether the fetal skin is punctured using ultrasound examination. Intra-amniotic pressure monitoring may aid in the prediction of adverse events and of the position of the needle tip; therefore, noninvasive monitoring of pressure changes could improve the safety of AI. Previous studies have also reported an increase in the intra-amniotic pressure with uterine contractions [11]. Therefore, it is important to consider uterine contractions while assessing the intra-amniotic pressure to prevent uterine contractions by tocolysis.

Although we did not observe adverse maternal and fetal events due to AI in this study, adverse events due to AI have been previously reported [7]. A previous study reported that the risk of complication increases with advanced GA and number of punctures [16]. Therefore, the indications for AI should be carefully selected, and only well-trained experts should perform AI.

As for the indication, we performed AI for the relief of umbilical cord compression or ultrasonographic accuracy in cases with oligohydramnios. We determined cases with umbilical cord compression according to the presence of fetal blood flow abnormalities or variable decelerations. We also performed AI for the relief of umbilical cord compression in cases without oligohydramnios. We speculated that umbilical compression with fetal blood flow abnormalities or variable decelerations on fetal heart rate monitoring could cause fetal blood flow stress due to the intermittent blood flow redistribution and lead to reductions in the fetal urine volume as a fetal compensation mechanism for fetal hypoxia, which could lead to a dangerous cycle of umbilical cord compression $[3,5]$. In the 15 cases with umbilical cord compression, fetal blood flow abnormalities tended to improve, or variable decelerations decreased after AI as in a previous report [5]. However, the efficacy has been not established. Although that does not influence this study, further prospective control studies are needed to clarify the efficacy of AI in such cases.

We acknowledge that there are several limitations of this study. The number of patients included was small; hence, our sample size limits the generalizability of our findings. However, this study aimed to evaluate the changes in intra-amniotic pressure following transabdominal AI not to evaluate the relationship between the pressure and complications and neonatal outcome. We could not perform subgroup comparisons in this study concerning the risk of complications related to AIs as no adverse maternal and fetal events were observed. Further data are required and detailed; thorough subgroup comparisons according to patients with and without complications are needed to clarify the relationship between the intra-amniotic pressure and the incidence of complications.

In the 3 cases with oligohydramnios, the fetus was punctured, which caused fetal skin edema. Although no severe complications related to fetal puncture were observed in this study, fetal injury with fetal puncture should be considered in studies regarding AIs. Intra-amniotic pressure monitoring, which can provide early detection of fetal puncture, might be effective to prevent severe complications related to fetal puncture. In addition, it is most important to check the position of the tip of the needle during AIs.

\section{Conclusion}

This study suggests that incident complications associated with increased intra-amniotic pressure are not likely to develop if the AFI after $\mathrm{AI}$ is within the normal range; however, we may need to consider individual uterine pressure tolerance. The risk of complications due to amniocentesis persists. Additionally, the intra-amniotic pressure may be effective in predicting the position of the tip of the needle in AI. Therefore, intra-amniotic pressure monitoring may be valuable for preventing adverse events during $\mathrm{AI}$ and increase the safety of the procedure because the technique is noninvasive and easily available.

\section{Acknowledgments}

We thank Takeshi Iwase and Hiroshi Soejima, medical engineers at Nagara Medical Center, for their assistance with the pressure measurements.

\section{Statement of Ethics}

Written informed consent was obtained from all study participants. The study protocol was approved by the Institutional Review Board of the Nagara Medical Center (18-6) and was carried out in accordance with the guidelines of the Declaration of Helsinki.

\section{Conflict of Interest Statement}

The authors have no conflicts of interest to declare.
Katsura/Takahashi/Iwagaki/Chiaki/Asai/ Koike/Murakami 


\section{Funding Sources}

There were no funding sources for this study.

\section{Author Contributions}

D.K. and Y.T. designed and planned the study; D.K. wrote and managed the manuscript; D.K., Y.T., S.I., R.C., K.A., M.M., S.Y., M.F., and T.M. performed the measurements; D.K. and Y.T. performed data analysis.

\section{Data Availability Statement}

The datasets during and/or analyzed during the current study are available from the corresponding author on reasonable request.

\section{References}

1 Hofmeyr GJ, Lawrie TA. Amnioinfusion for potential or suspected umbilical cord compression in labour. Cochrane Database Syst Rev. 2012 Jan;1(1):CD000013.

2 Dad N, Abushama M, Konje JC, Ahmed B. What is the role of amnioinfusion in modern day obstetrics? J Matern Fetal Neonatal Med. 2016 Sep;29(17):2823-7.

3 Takahashi Y, Iwagaki S, Chiaki R, Iwasa T, Takenaka M, Kawabata I, et al. Amnioinfusion before 26 weeks' gestation for severe fetal growth restriction with oligohydramnios: Preliminary Pilot Study. J Obstet Gynaecol Res. 2014 Mar;40(3):677-85.

4 KozinszkyZ, PásztorN, VanyaM, Sikovanyecz J, Pál A. Management of severe idiopathic oligohydramnios: is antepartum transabdominal amnioinfusion really a treatment option? J Matern Fetal Neonatal Med. 2013 Mar; 26(4):383-7.

5 Katsura D, Takahashi Y, Iwagaki S, Chiaki R, Asai K, Koike M, et al. Amnioinfusion for variable decelerations caused by umbilical cord compression without oligohydramnios but with the sandwich sign as an early marker of deterioration. J Obstet Gynaecol. 2019 Jan; 39(1):49-53.

6 Fisk NM, Ronderos-Dumit D, Soliani A, Nicolini U, Vaughan J, Rodeck CH. Diagnostic and therapeutic transabdominal amnioinfusion in oligohydramnios. Obstet Gynecol. 1991 Aug;78(2):270-8.
7 National Institute for Health and Clinical Excellence. Interventional procedures overview of therapeutic amnioinfusion for oligohydramnios during pregnancy (excluding labour). The National Institute for Health and Clinical Excellence (NICE); 2006 [cited 2018 Oct 30]. Available from: https://www.nice. org.uk/guidance/ipg192/resources/therapeutic amnioinfusion-for-oligohydramnios-during-pregnancy-excluding - labourpdf-1899863518635205.

8 Guzman ER, Vintzileos A, Benito C, Houlihan C, Waldron R, Egan S. Effects of therapeutic amniocentesis on uterine and umbilical artery velocimetry in cases of severe symptomatic polyhydramnios. J Matern Fetal Med. 1996 Nov-Dec;5(6):299-304.

9 Katsura D, Takahashi Y, Iwagaki S, Chiaki R, Asai K, Koike M, et al. Relationship between higher intra-amniotic pressures in polyhydramnios and maternal symptoms. Eur J Obstet Gynecol Reprod Biol. 2019 Apr;235:62-5.

10 Katsura D, Takahashi Y, Iwagaki S, Chiaki R, Asai K, Koike M, et al. Intra-amniotic pressure of twin-to-twin transfusion syndrome. Fetal Diagn Ther. 2018;44(2):160.
11 Katsura D, Takahashi Y, Iwagaki S, Chiaki R, Asai K, Koike M, et al. Changes in intra-amniotic, fetal intrathoracic, and intraperitoneal pressures with uterine contraction: a report of three cases. Case Rep Obstet Gynecol. 2018 Sep;2018:4281528.

12 Phelan JP, Smith CV, Broussard P, Small M. Amniotic fluid volume assessment with the four-quadrant technique at 36-42 weeks' gestation. J Reprod Med. 1987 Jul;32(7):540-2.

13 Chamberlain PF, Manning FA, Morrison I, Harman CR, Lange IR. Ultrasound evaluation of amniotic fluid volume. I. The relationship of marginal and decreased amniotic fluid volumes to perinatal outcome. Am J Obstet Gynecol. 1984 Oct;150(3):245-9.

14 Kanda Y. Investigation of the freely available easy-to-use software "EZR" for medical statistics. Bone Marrow Transplant. 2013 Mar; 48(3):452-8.

15 Brace RA, Wolf EJ. Normal amniotic fluid volume changes throughout pregnancy. Am J Obstet Gynecol. 1989 Aug;161(2):382-8.

16 Nassar AH, Martin D, González-Quintero VH, Gómez-Marín O, Salman F, Gutierrez A, et al. Genetic amniocentesis complications: is the incidence overrated? Gynecol Obstet Invest. 2004;58(2):100-4. 